\title{
Fumarsäure und Maleinsäure und deren Salze.
}

In zwei interessanten Abhandlungen, wovon die erste von $\mathrm{Ri}$ eckher und die zweite von $B$ üchner in dem Laboratorium zu Giessen verfasst ist, sind die Resultate sehr ausführlicher Untersuchungen über die Constitution dieser Säuren und ihrer Verbindungen niedergelegt. Es ergiebt sich daraus, dass diese beiden von Pelo uze bei der trocknen Destillation der krystallisirten Aepfelsäure zuerst beobachteten und von ihm als isomer erkannten Säuren allerdings gleiche procentische Zusammensetzung haben; jedoch abgesehen von ihren an und für sich sehr äbweichenden Eigenschaften auch bei der Verbindung mit Basen sich wesentlich verschieden verhalten und die eine als einbasische, die andere als $\mathrm{zw}$ eibasische Säure auftritt.

Winkler fand in der Fumaria officinalis eine eigenthümliche Säure, welche er Fumarsäure nannte und D emarcay wies später nach, dass dieselbe mit der Parameleinsäure (Pelouze) identisch sei. - Zur leichteren Uebersicht sind hier die Eigenschaften dieser Säure und die der Maleinsaure neben einander gestellt.

Die Fumarsäure krystallisirt in breiten, zusammengehäuften dünnen Säulen, bald mit rhömboedrischer, bald mit sechsseitiger Basis, häufig auch in kleinen Blättchen von undeutlicher Form.

Die Maleinsäure bildet schiefe, rhombische Säulen, durch Octaederflächen abgestumpft. Beim freiwilligen Verdunsten der wässerigen Lösung efflorescirt die Säure an den Wandungen der Gefässe.

Die Furmarsäure ist in 200 Theilen kalten Wassers löslich; in Weingeist und Aether löslich, desgleichen in Salpetersäure von 1,40 spec. Gew., welche auch im Kochen dieselbe nicht zersetzt. Concentrirte Schwefelsäure zersetzt sie in der Kälte nicht; bei erhöheter Temperatur jedoch unter Entwickelung von schwefliger Säure.

Die Maleinsäure ist leicht löslich in Wasser, Alkohol und Aether.

Die Fumarsäure hat einen rein sauern Geschmack.

Die Maleinsäure schmeckt heftig sauer, hintennach kratzend, zusammenziehend, metallisch.

Die Fumarsäure schmilzt sehr schwierig, verflüchtigt sich erst bei einer Temperatur über $200^{\circ}$. Wird dieselbe sublimirt, so verwandelt sie sich in Maleinsäure.

Die Maleinsäure schmilzt bei $130^{\circ}$ und kocht bei $160^{\circ}$ Einer raschen Destillation unterworfen zerlegt sich das 
Maleinsäurehydrat in eine weisse bei $57^{\circ}$ schmelzende und bei $176^{\circ}$ siedende fluichtige Materie, welche wasserfreie Maleinsäure ist. - Längere Zeit geschmolzen, geht die flüssige Masse des Maleinsäurehydrats in einen festen krystallinischen Brei von reinem Fumarsäurehydrat uiber.

Kalkwasser fällt beide Säuren nicht.

Barytwasser giebt mit der Fumarsäure keine Trübung; der krystallinische Absatz, welcher sich aus concentrirten Lösungen nach und nach abscheidet, ist in der Säure und im Fällungsmittel schwer löslich.

Barytwasser giebt mit der Maleinsäure einen weissen Niederschlag; nach einigen Minuten verwandelt sich derselbe in glänzende Blättchen, die in der Säure und dem Fällungsmittel leicht löslich sind.

Chlorcalcium giebt mit beiden Säuren keine Reaction.

Essigsaures Bleioxyd erzeugt mit der Fumarsäure einen weissen voluminösen, nach und nach zusammensinternden Niederschlag aus mässig concentrirten Lösungen. Aus verdünnter und kochendheisser Flüssigkeit krystallisirt ein Theil in glänzenden Blättchen.

Mit der Maleinsäure entsteht ein weisser Niederschlag in verdünnter Lösung, der nach einigen Minuten glimmerartig erscheint. In concentrirter Flüssigkeit gesleht der Niederschlag zu einer kleisterartigen Masse.

Salpetersaures Silberoxyd bildet in der vorher mit Ammoniak neutralisirten Fumarsäure einen weissen, selbst nach 24. Stunden sich nicht verändernden Niederschlag.

Mit der Maleinsäure entsteht unter gleichen Umständen ein weisser Niederschlag, der nach einiger Zeit in farblose durchsichtige Krystalle sich verändert.

Elementarausammensetzung beider Säuren :

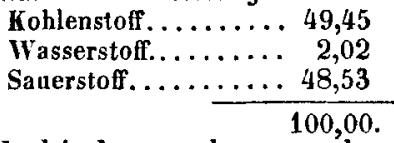

Da aus den Verbindungen hervorgeht, dass die Fumarsäure eine einbasische, die Maleinsäure eine zweibasische Säure ist, und wenn das Aequivalent des Wasserstoffs nicht mehr wie früher mit $\mathrm{H}^{2}$, sondern mit $\mathrm{H}$ bezeichnet wird, so ist die Formel für:

$$
\begin{aligned}
& \text { Fumarsäure }=\mathrm{C}^{4}+\mathrm{H}+\mathrm{O}^{3}=\overrightarrow{\mathrm{Fu}} \\
& \text { Atomg. } 615,88 . \\
& \text { Maleinsäure }=\mathrm{C}^{8}+\mathrm{H}^{2}+\mathrm{O}^{6}=\overrightarrow{\mathrm{Ma}} \text {. } \\
& \text { Atomg. 1236,646. }
\end{aligned}
$$$$
\text { Ilydrat }=\mathrm{HO}+\overrightarrow{\mathrm{Fu}} \text {. }
$$ 


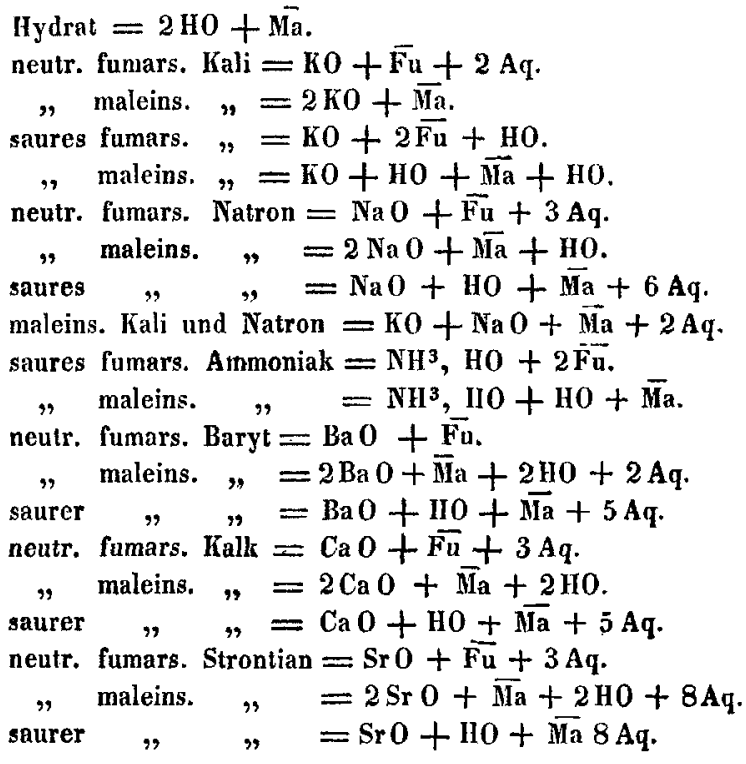

neutr. fumars. Magnesia. Das luftrockne Salz hat die Formel $=\mathrm{MgO}+\overrightarrow{\mathrm{Fu}}+4 \mathrm{Aq}$. Bei $100^{\circ}$ getrocknet behält es noch

2 At. Wasser zurück, die es bei $200^{\circ}$ fahren lässt.

neutr. maleins. Magnesia $=2 \mathrm{MgO}+\overrightarrow{\mathrm{Ma}}+2 \mathrm{HO}+6 \mathrm{Aq}$.

saure $" \quad, \quad=M g 0 \pm \overline{M a}+7$ Aq.

fumars. Cobaltoxydul $=\mathrm{CoO}+\widehat{\mathrm{F}_{\mathrm{u}}}+3 \mathrm{Aq}$.

$$
\text { Nickeloxyd }=\mathrm{NiO}+\overline{\mathrm{Fu}}+3 \mathrm{Aq} \text {. }
$$

maleins.,$\quad=2 \mathrm{NiO}+\overline{\mathrm{Ma}}+2 \mathrm{HO}$.

funars. Manganoxydul $=\mathrm{MnO}+\widetilde{\mathrm{Fu}}+3 \mathrm{Aq}$.

$" \quad$ Eisenoxyd $=\mathrm{Fe}^{2} \mathrm{O}^{3}+\mathrm{Fu}$.

$" \quad$ Zinkoxyd :

man kann 2 krystallisirte Salze erhalten von folgender Zusammensetzung: 2 ( $\mathrm{ZnO}+\mathrm{Fu})+3 \mathrm{Aq}$.

$$
\mathrm{ZnO}+\overline{\mathrm{Fu}} \pm \mathrm{Aq} \text {. }
$$

maleins. Zinlsoxyd $=2 \mathrm{Zn}+\overline{\mathrm{Ma}}+4 \mathrm{HO}$.

neutr. funars. Bleioxyd $=\mathrm{PbO}+\overline{\mathrm{Fu}_{\mathrm{u}}}+2$ Aq. und

$$
\mathrm{PbO}+\mathrm{Fu}+3 \mathrm{Aq} \text {. }
$$

maleins. Bleioxyd $=2 \mathrm{PbO}+\overline{\mathrm{Ma}}+2 \mathrm{HO}+4 \mathrm{Aq}$.

basisch fumars. Bleioxyd $=3 \mathrm{PbO}+\overline{\mathrm{Fu}}$ und

$$
3 \mathrm{~Pb} 0+2 \overline{\mathrm{Fu}} \text {. }
$$

fumars. Kupferoxyd $=\mathrm{CuO}+\overline{\mathrm{Fu}}+\mathrm{Aq}$.

maleins. $"=2 \mathrm{CuO}+\overrightarrow{\mathrm{Ma}}+2 \mathrm{HO}$. 


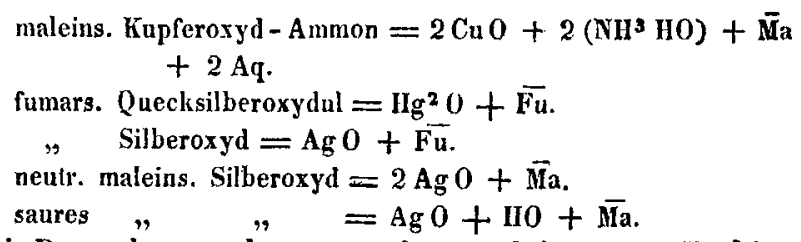

Bei Betrachtung der neutralen maleinsauren Verbindungen erscheint es bei den meisten derselben als eigenthümlich, dass die 2 At. Hydratwasser der Säure auch in die Verbindung mit übergegangen sind, welches in der Regel bei den zweibasischen Säuren nicht der Fall ist; jedoch deutet dieses nach der Ansicht des Dr. Bü chner darauf hin, dass dieses Hydratwasser vertreten werden kann durch andere neutrale maleinsaure Verbindungen, wovon die Existenz des maleinsauren Kupferoxyd-Ammoniumoxyds ein Beispiel giebt.

Dass die Maleinsäure eine zweibasische Säure ist, geht aus dem aus Kali und Natron gebildeten Doppelsalze, so wie aus den sauren Erdsalzen und dem sauren Silbersalze auf unzweideutige Weise hervor. Eine besondere und bis jetzt noch unerklärte Anomalie zeigt aber die saure maleinsaure Magnesia, in welcher beide Atome Hydratwasser durch 1 Atom Magnesia ersetzt sind. Die 7 Atome Wasser treten bei $100^{\circ} \mathrm{C}$ aus, gehören desshalb nicht zur Constitution des Salzes und können nur als Krystallwasser betrachtet werden. (Annal. d. Chem. u. Pharm. Bd. 49. p. 31-91.)

Herzog.

\section{Untersuchungen über verschiedene gerbstoffhaltige Pflanzen von Stenh ouse.}

Schwarzer und grüner Thee. Nach Mulder stammen beide Sorten von derselben Pflanze und nur die verschiedene Zeit der Einsammlung und das schärfere Trocknen des erstern bewirken die Unterschiede. Aus der durch die Einwirkung der Hitze bedingten theilweisen Verwandlung des ätherischen Oels in Harz geht der Grund hervor, aus welchem wässeriger Aufguss von schwarzem Thee beim Erkalten sich truibt, während der von grünem Thee klar bleibt.

Die Aufgüsse beider Sorten geben mit Eisenvitriol schwärzlich olivengriune, beim Stehen schwarz werdende Niederschläge. Beide Aufgüsse geben beim Bindampfen und Destilliren Theile in Krystallen, während das empyreumatische Destillat Pyrogallussäure liefert. Zur Isolirung 